\title{
Three-dimensional Terrain Correcting for Detection of WSN Space Coverage Hole
}

\author{
Shen Xian-hao, Li Jun and Nai He \\ College of Information Science and Engineering, Guilin University of Technology, \\ Guilin 541004, China \\ lyj_sxh@sina.com; leejun19901113@163.com
}

\begin{abstract}
When wireless sensor networks using spatial random deployment, disturbed three-dimensional topography of factors can lead to the generation of the coverage hole. In order to effectively detect the coverage holes in the target area, in this paper, we propose a method for detecting space coverage holes in sensor networks based on three-dimensional terrain correction. Firstly using Delaunay triangulation subdivides network coverage and calculates the Delaunay triangle circumscribed circle, secondly using the cluster grouping method to build the cover blind area boundary. Thirdly according to the principles of the terrain correction, we calculate the actual detection radius, after terrain correction ultimately, obtain minimum boundaries of coverage holes. Simulation results show that the detection method can effectively detect coverage holes under the three-dimensional terrain. For the undulating obviously terrain, it also has some adaptability.
\end{abstract}

Keywords: Space Coverage Holes; Three-dimensional Terrain Correction; Delaunay Triangulation; Circumscribed Circle;

\section{Introduction}

Wireless sensor network (WSN) is composed of a large number of cheap micro-sensor nodes, randomly deployed in a monitored area, the network system is formed by wireless communication and self-organization [1]. Coverage performance is a key indicator to measure the quality of service in wireless sensor networks. Improving continuously coverage performance in recent years become a major research focus. Repairing coverage holes is one of the important research content. In the target area use randomly deployment of sensor nodes. Resulting in some monitored area have no sensor nodes, which appeared coverage holes, seriously affecting network performance. In addition, with running of the network, energy depletion of sensor network nodes will also lead to the formation of the coverage hole. Therefore, when the coverage holes appear in the network, they should be detected immediately. In order to maintain the integrity and reliability of wireless sensor networks [2].

For the current coverage holes of wireless sensor network, many researchers have proposed some solutions. Hwa Chun $\mathrm{M}$ et al. [3] in this paper a computational geometry approach based distributed hole detection protocol is designed to find out the coverage holes in a post deployment scenario. An efficient geometric method with proper theoretical basis is used to detect the coverage holes of the wireless sensor network. Dai, Chen and Zhou [4] proposed coverage hole detection algorithm based on Voronoi diagram in wireless sensor network. The location information of sensor nodes was used to build the Voronoi diagram for the monitored area. Then the distances between a sensor node and the vertex or edges of the corresponding Voronoi cell were calculated to decide the existence of coverage holes and identify the border nodes. Gao, Wang and Feng et al. [5] proposed a geometry-based distributed coverage holes discovery algorithm. The 
algorithm judges existence of coverage blind spots under the guidance of relevant theory of geometric graphics. Xing, Duan and Fan [6] proposed a distributed WSNs coverage holes discovery algorithm based on polar coordinate, the algorithm uses polar coordinate to express relationship between nodes, through geometric algorithm to detect whether there is blind spot of coverage in WSNs. Li and Chen [7] proposed coverage holes detection algorithm in wireless sensor networks. The main idea of this algorithm is calculating boundary arcs and boundary nodes of coverage holes, according to coverage holes surrounded by boundary arcs. Above the several algorithms are all the coverage hole detection method on the two dimensional plane, it does not apply to the three-dimensional terrain. Liu and $\mathrm{Fu}$ [8] proposed a topographic correction algorithm based on the slope information in digital elevation maps to detect trap holes in rugged terrain by constructing directional perception model for the sensor nodes. However this method only applies to slope gently of undulating terrain, does not apply to fluctuate obviously on terrain three-dimensional.

According to above theoretical research, this paper focuses on a more reliable three dimensional undulating terrain, proposed detection algorithm of coverage hole in WSN based on three-dimensional terrain correction. The algorithm can effectively detect the coverage holes in the target area.

\section{Formation of Coverage Holes}

In the research of wireless sensor networks, coverage is one of the basic problems. It affects the productivity and quality of WSN. The core of the coverage is the sensor nodes by collecting information to achieve the target area monitoring and perception. Through the rational deployment of sensor nodes in the network, at the request of guaranteed network quality of service, maximum area to be covered. Typically, the sensor nodes are randomly deployed in the target area. However this way is hard to a reasonable location of the plurality of sensor nodes, resulting in network coverage is not reasonable, eventually forming the perceived overlap and coverage holes [9]. As shown in Figure 1, a two-dimensional plane of covering research methods cannot be applied to three dimensional. After the random deployment of sensor nodes, since the characteristics of three dimensional undulating terrain, it can lead to the generation of the coverage hole [10].

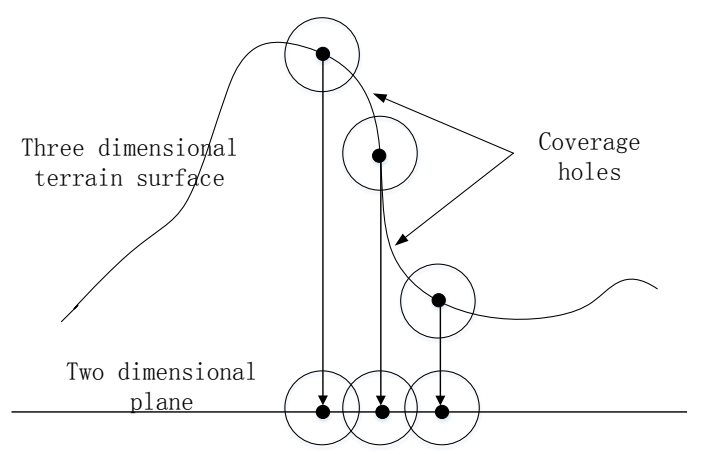

\section{Figure 1. Section Diagram of Coverage Hole}

Projection of sensor nodes achieve full coverage on a two dimensional plane. However, sensor nodes can only be placed on the ground surface. Then coverage holes still exist on the surface of the three-dimensional terrain. 


\section{Coverage Hole Detection Algorithm and Terrain Correction}

Sensor nodes are randomly deployed in the target area, it will have coverage holes. We construct the Delaunay triangulation of the WSN, and compute the circumcircle of the Delaunay triangle. Assuming the radius of sensor node is $r$. The radius of the circumcircle is R. The length of common side of two Delaunay triangles is d. Detection algorithm is calculated as follows:

Step 1 Construct the Delaunay triangulation of the WSN.

Step 2 Compute the circumcircle of the Delaunay triangle. Compare radius of sensor node and circumcircle. If $\mathrm{R}>\mathrm{r}$, then there must be some coverage holes, store this Delaunay triangle and circumcircle, otherwise abandon it.

Step 3 Calculate the length $\mathrm{d}$ of common side of two adjacent Delaunay triangles. If $\mathrm{d}>2 \mathrm{r}$ or the common side is not intersect the line segment between the centers of circumcircle of the two triangles, then cluster the circumcircle of the two triangles into one coverage hole group. And every group will have coverage holes.

Step 4 Plot the boundaries of coverage holes of every group by minimum polygon method.

Step 5 By determination principle of false boundary nodes in Figure 8, we remove the false boundary nodes from the corresponding boundary nodes group. we do the verification for each boundary nodes group until no false boundary nodes. In this way, we can obtain a precisely boundary of every group.

Step 6 Because of the characteristics of three-dimensional undulating terrain, the actual coverage area will be smaller. After terrain correction, coverage area of sensor nodes is elliptic on two-dimensional plane. Using slope and aspect to calculate actual radius, and obtain the corrected boundary by coverage hole detection algorithm.

\section{Network Model and Measurement}

\subsection{Establishing the Unit Ball Perception Model}

we assume the target area is a convex surface $\mathrm{S}$ in three dimensional space. In the Cartesian coordinate system, $\mathrm{S}$ can be expressed as a single value function $z=h(x, y)$. If and only if the function is $z=c$ where $\mathrm{c}$ is a constant, $\mathrm{S}$ is a plane. A sensor $s_{i}$ is placed on S. If the coordinates of $s_{i}$ satisfy the equation of $\mathrm{S}$, then expressed as $s_{i} \in S$. This paper use a unit ball perception model. We assume that each sensor has the same perception radius $r$ in three dimensional space. Sensors can perceive and detect events within its own perception range. Therefore perception area forms a ball of radius $r$ centered at $s_{i}$ in three dimensional space. Two dimensional plane of unit ball model is shown as Figure 2.

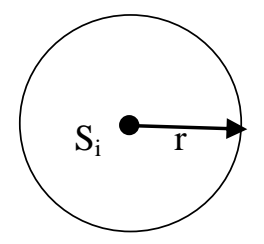

Figure 2. Two Dimensional Plane of Unit Ball Model

\subsection{Sensor Deployment Model}

Sensor nodes are randomly deployed in the target area [11]. We consider the deployment of surface Poisson point distribution model. Assume $\mathrm{n}$ sensor nodes are distributed uniformly, area of the target region $S \rightarrow \infty$. Then we can get the parameter of the Poisson distribution [12]. 


$$
\lambda=\frac{n}{S}
$$

The probability that there are $\mathrm{m}$ sensors lie in a set $\mathrm{G}$ is calculated as follows:

$$
\frac{(\lambda G)^{m}}{m !} e^{-\lambda G}
$$

\subsection{Definition of Slope and Aspect}

Slope is the degree of terrain surface steepness. Usually the ratio of vertical height and horizontal distance called the slope. Aspect is slope surface normal line in the direction of the projection on a horizontal plane. It is also the fastest decline in the elevation direction. The actual coverage of single sensor node on the three dimensional surfaces can be equivalent to the plane. As shown in Figure 3., slope and aspect represented by AB line segment in the Cartesian coordinate system.

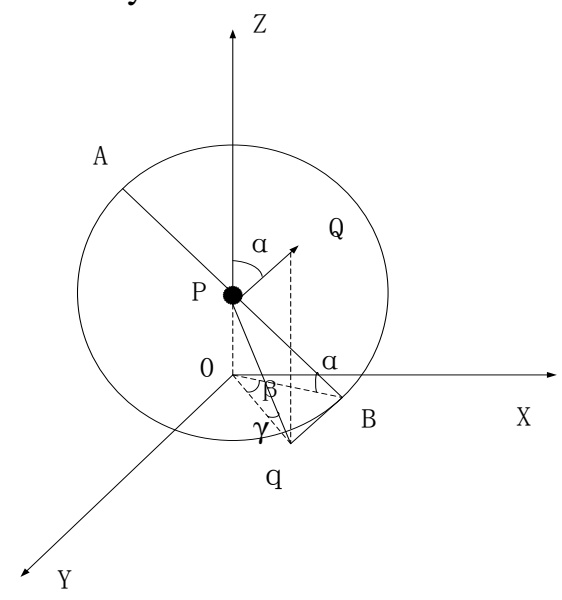

Figure 3. Slope and Aspect

Where $\mathrm{P}$ on the three-dimensional convex curved surface. The normal line of $\mathrm{AB}$ line segment is PQ. The $q$ is the projection of $Q$ in the $X Y$ plane. The Oq is projection of PQ in the XY plane. The $\alpha$ is the angle between $Z$ axis and PQ. So slope can be expressed as $\mathrm{I}=\tan \alpha$. Aspect $\beta$ is the angle between $\mathrm{Oq}$ and OB. The slope in the $\beta$ direction can be expressed as $\tan \gamma$.

Through GIS software extracting elevation data and slope information from the DEM [13]. On the surface $z=h(x, y), p(x, y)$ the direction of the gradient is calculated as follows:

$$
\operatorname{grad} \mathrm{f}(\mathrm{x}, \mathrm{y})=\frac{\partial f}{\partial x} \vec{i}+\frac{\partial f}{\partial y} \vec{j}
$$

Where $\frac{\partial f}{\partial x}$ and $\frac{\partial f}{\partial y}$ respectively the partial derivative of the $\mathrm{x}$ and $\mathrm{y}$ directions. The $\mathrm{i}$ and $\mathrm{j}$ are the unit vectors.

The direction of gradient mode is calculated as follows:

$$
\boldsymbol{I}=\frac{|P O|}{|O B|}=\mathrm{t} \mathbf{a} \boldsymbol{\alpha} \boldsymbol{x}=\sqrt{\left(\frac{\partial f}{\partial x}\right)^{2}+\left(\frac{\partial f}{\partial y}\right)^{2}}
$$

The slope $\mathrm{G}$ in the $\beta$ direction can be calculated as follows:

$$
G=\tan \gamma=\frac{|P O|}{|O q|}
$$

Using Eq. $(4,5), \mathrm{G}$ is also defined as follows:

(6)

$$
G=I c \text { o } \beta
$$


On the three-dimensional undulating terrain, the relationship between the actual radius $r$ ' along the direction of $\beta$ and the ideal radius $r$ can be expressed as follows:

$$
r^{\prime}=r \cos
$$

Using Eq. $(5,6,7)$, the actual radius $r$ ' is also calculated as follows:

$$
r^{\prime}=r \cos (\arctan (I \cos \beta))
$$

\subsection{Terrain Correction Principle}

Random deployed 50 sensor nodes in the area of $100 \times 100 \mathrm{~m}$ [14]. The Figure 4 shows the 120-200 contours.

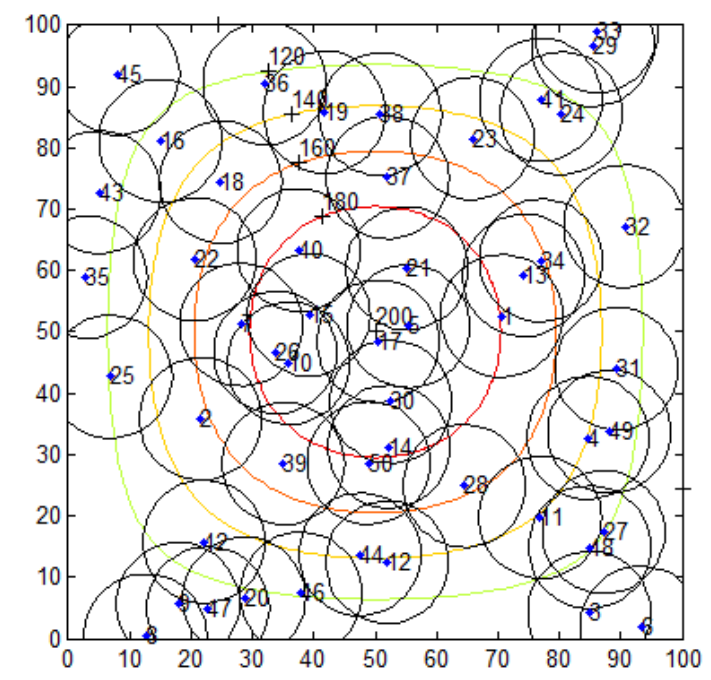

Figure 4. Terrain Correction Principle

According to sensor node coordinates calculate the slope and aspect angle of each node. Along the aspect direction of difference between two contours is the height difference $\Delta h$. The distance between the two contour lines is $\Delta d$. Then slope I can be calculated as follows:

$$
I=\frac{\Delta h}{\Delta d}
$$

Using Eq. (8) calculates the actual radius $r$ '. Eventually calculated the elliptic projection of each sensor node on a two-dimensional plane. To achieve the purpose of terrain correction.

\subsection{Delaunay Triangulation}

Central point for sensor nodes as vertices, Delaunay Triangulation the target area. Assumed $\mathrm{V}$ is a finite point set on two-dimensional real numbers, e is a closed line segment. And $\mathrm{E}$ is the set of e. Then a triangulation $T=(V, E)$ of $\mathrm{V}$ is a plane graph $\mathrm{G}$. The plane graph $\mathrm{G}$ meets the three conditions:

(1) Except the endpoint, edge of the plane G does not contain any other points in point set $\mathrm{V}$.

(2) Without intersecting edges.

(3) All planes are triangles in plane graph G. And the collection of all the triangles is the convex hull of point set $\mathrm{V}$.

If there is a circle through $\mathrm{a}$ and $\mathrm{b}$, and does not contain other points within the circle. Then ab edge is called Delaunay edge. If a triangulation $\mathrm{T}$ only contains Delaunay edge, then the triangulation called Delaunay triangulation[15]. 


\subsection{Circumcircle circumscribed circle}

The circumcircle of each Delaunay triangles does not include any vertex of other Delaunay triangles[16]. Assume that a, b, c are three sides of Delaunay triangle. $\mathrm{S}$ is the area of the triangle. $(\mathrm{x}, \mathrm{y})$ is the center of circumcircle. As shown in Figure 5.

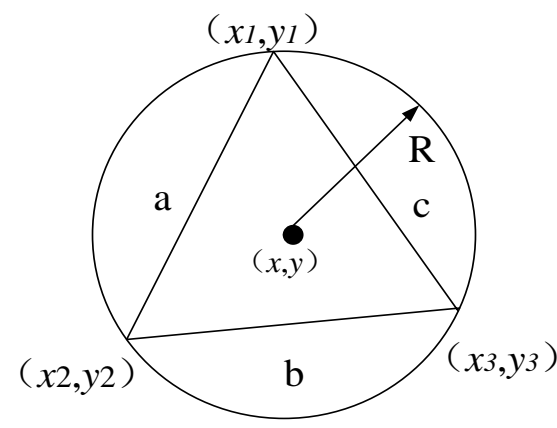

Figure 5. Circumcircle

Circumcircle radius $\mathrm{R}$ is expressed as:

$$
R=a b c / 4 S
$$

Assuming that the coordinates of the three sides are (x1, y1), (x2, y2) and (x3, y3). So Circumcircle center coordinates $(\mathrm{x}, \mathrm{y})$ can be expressed as:

$$
\begin{gathered}
x=\frac{\left(x_{1}^{2}-x_{2}^{2}+y_{1}^{2}-y_{2}\right)^{2}\left(y_{1}-y_{3}-\left(x_{1}-z_{3}^{2}+\hat{y}_{1}-y_{3}^{2}(x)\right.\right.}{\left.2\left(y_{1}-y_{3}\right)\left(x_{1}-x_{2}+2 x_{1}-y_{2}\right) x_{-} x_{3}\right)} \\
y=\frac{(11)}{2\left(x_{1}^{2}-x_{2}^{2}+y_{1}^{2}-y_{2}\right)^{2}\left(x_{1}-x_{3}\right)\left(x_{1}-y_{1}^{2}-y_{2}^{2}+2 x_{3}^{2}+x_{2}^{2}\right) x_{1}^{2}-y_{3}^{2}(x)}
\end{gathered}
$$

\subsection{Boundary Nodes and False Boundary Nodes}

The nodes on the boundaries that encircle the coverage holes are defined as boundary nodes[17]. Generally, the nodes on the Delaunay triangles that lie on the boundaries of coverage holes can be regarded as boundary nodes. However, these boundaries exist false boundary node. As shown in Figure 6 and Figure 7, the Delaunay triangle with vertices S1, S2 and S3 is on the boundary of a coverage hole.

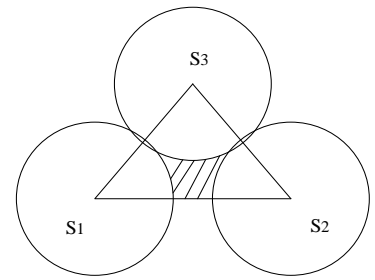

Figure 6. Boundary Nodes

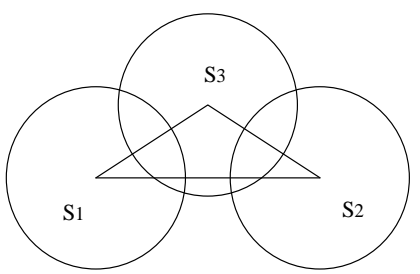

Figure 7. False Boundary Nodes

In Figure 6, some region inside the triangle is not covered by any node, shown as the shaded area. Accordingly, S1, S2 and S3 are boundary nodes. However, in Figure 7, the coverage hole is located outside of the triangle, so S1, S2 are boundary nodes. The S3 in Figure 7 is not right on the boundary of the coverage hole, which is called false boundary node.

Theorem 1. Consider a triangle formed by three nodes, S1, S2 and S3. The perception radius of nodes is $\mathrm{r}$. The center $O_{c}$ of circumcircle is outside the triangle. The radius of 
circumcircle is R. As shown in Figure 8, the perpendicular bisector of S1S3 intersects $\mathrm{S} 1 \mathrm{~S} 2$ at $\mathrm{O} 1$, and the perpendicular bisector of $\mathrm{S} 2 \mathrm{~S} 3$ intersects $\mathrm{S} 1 \mathrm{~S} 2$ at $\mathrm{O} 2, \mathrm{~S} 3 \mathrm{O} 3$ is perpendicular to S1S2 at O3. If $\mathrm{S}_{3} \mathrm{O}_{1}>r$ or $\mathrm{S}_{3} \mathrm{O}_{2}>r$, some coverage holes inside the $\Delta S_{1} S_{2} S_{3}$. If $S_{3} O_{1}>r, S_{3} O_{2}>r$ and $S_{3} O_{3}<=r$, then two separated coverage holes in the $\Delta S_{1} S_{2} S_{3}$.

For a Delaunay triangle whose center of circumcircle is outside the triangle and $\mathrm{R}>\mathrm{r}$, according to Theorem 1, we can estimate whether the triangle is fully covered by sensors. If there is no uncovered region inside the triangle, the node that is not on the longest side is a false boundary node of coverage holes. Otherwise, if the triangle contains some uncovered region, three nodes belong to the boundary nodes.

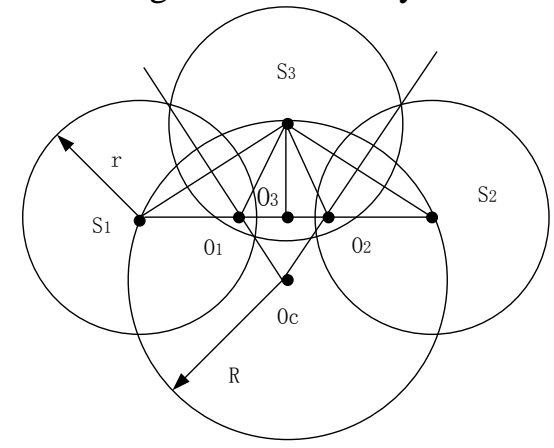

Figure 8. Determination Principle of False Boundary Nodes

\section{Experimental Studies}

\subsection{Surface Generation}

Thesis uses MATLAB 2012b simulation platform for simulation. In order to represent surface coverage performance, we use $z=h(x, y)$ to represent the generated surface [18].

$$
z=100+100 \sin (C \pi x / 100) \sin (C \pi y / 100)
$$

where $\mathrm{x}, \mathrm{y} \in[0,100] \mathrm{m}, \mathrm{C}=1,2,3$ to generate three surfaces with $1,4,9$ peaks and valleys in $[0,100] \times[0,100] \mathrm{m}$. Figure 9 gives the contours of these surfaces.
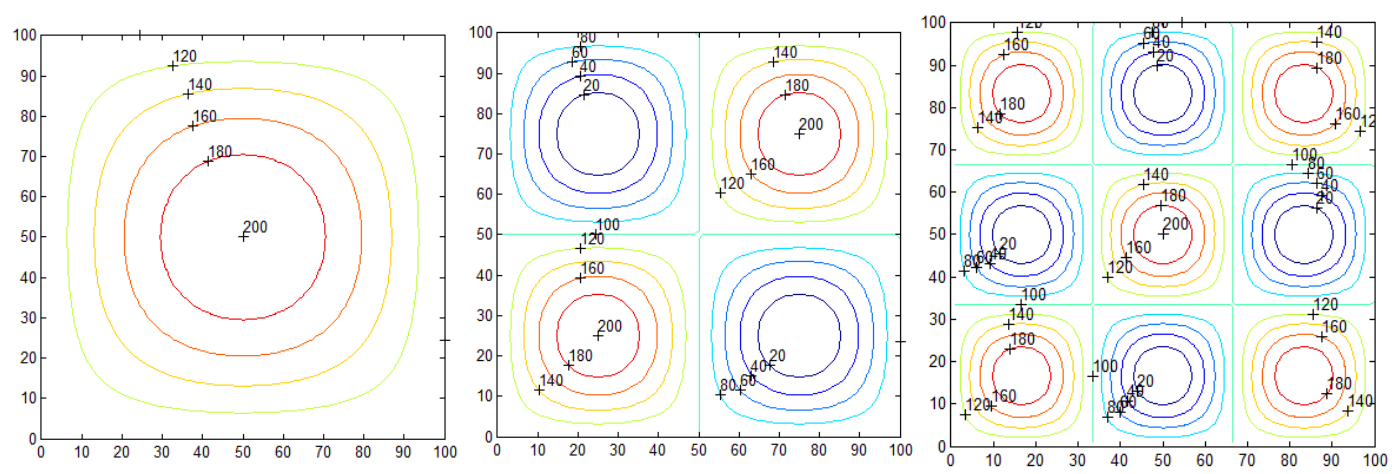

Figure 9. Contours of Three Surfaces 


\subsection{Simulation Results}

Assume the number of sensor nodes deployed randomly $\mathrm{N}=50$, the ideal detection radius of nodes $\mathrm{r}=10 \mathrm{~m}$, in the region $[0,100] \times[0,100] \mathrm{m}$. The circumcircle radius of each Delaunay triangle is R. Simulation parameters as shown in Table 1. Construct Delaunay triangulation of 50 sensor nodes, we can obtain a number of Delaunay triangles. As shown in Figure 10, Where the circular area represents the coverage area of the node.

Table 1. Simulation Parameters

\begin{tabular}{lc}
\hline Simulation parameters & value \\
\hline Network range $(\mathrm{m} 2)$ & $100 \times 100$ \\
Number of sensor nodes & 50 \\
Detection radius $(\mathrm{m})$ & 10 \\
Dispenser way & random \\
\hline
\end{tabular}

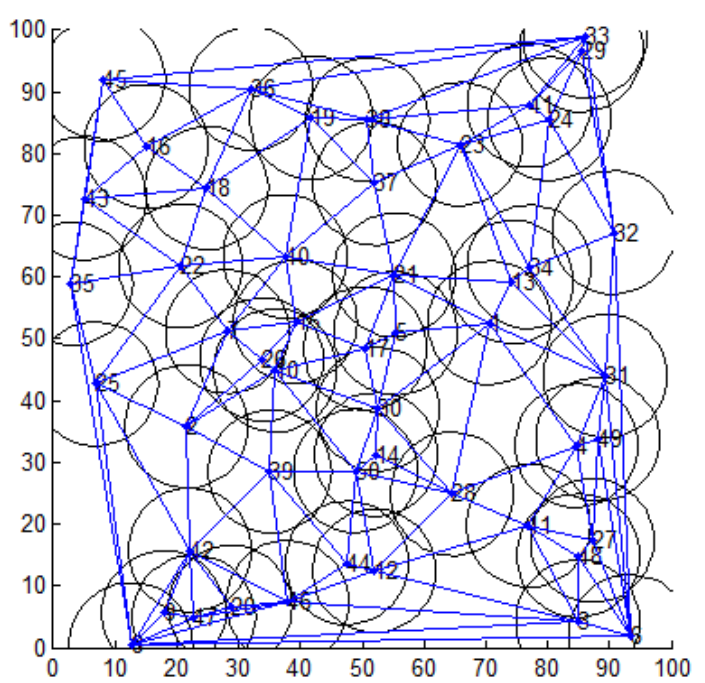

Figure 10. Delaunay Triangulation

According to Delaunay triangle vertex coordinates, we can be determined circumcircle radius $\mathrm{R}$ and circumcircle center of each triangle. As shown in Figure 11, if $\mathrm{R}>\mathrm{r}$, the target area has coverage holes.

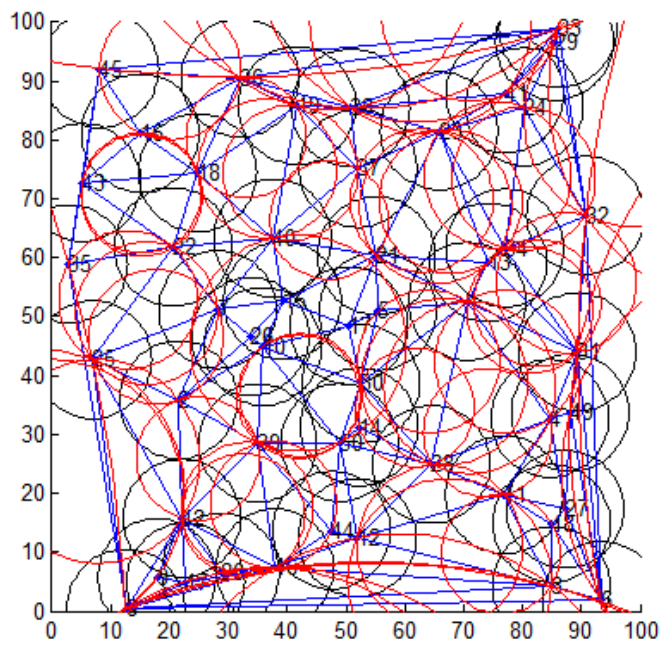

Figure 11. Circumcircle of $R>r$

According to coverage holes boundary detection algorithm, we cluster eligible 
Delaunay triangles. Plot the boundaries of coverage holes of every group by minimum polygon method. As shown in Figure 12.

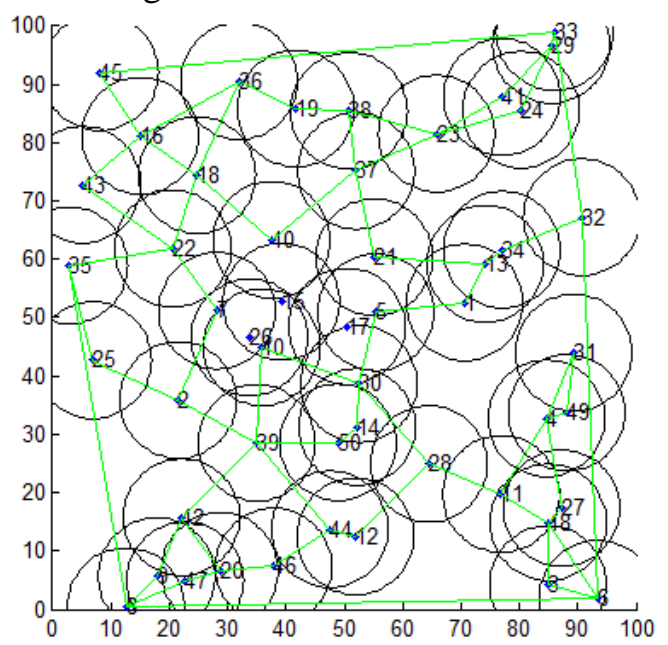

\section{Figure 12. Boundaries of Coverage Holes}

Removed the false boundary nodes, we can obtain the boundary nodes for every coverage hole precisely. Then we can obtain the improving boundaries. As shown in Figure 13.

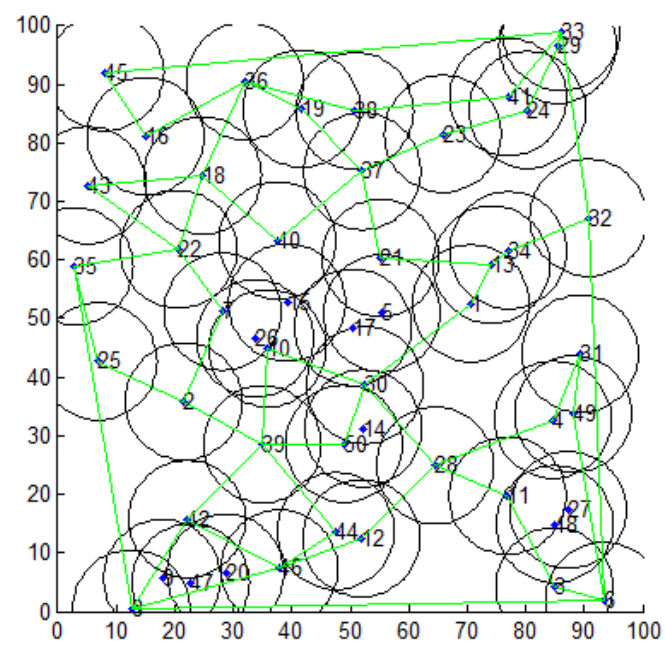

Figure 13. Improving Boundaries

According to the degree of undulating terrain, as shown in Table 2, we set three terrain. After the three-dimensional undulating terrain correction, we can obtain coverage hole boundary precisely.

Table 2. Terrain Parameters

\begin{tabular}{lccc}
\hline Terrain Type & Terrain I & Terrain II & Terrain III \\
\hline Network range $(\mathrm{m} 2)$ & $100 \times 100$ & $100 \times 100$ & $100 \times 100$ \\
Peaks and valleys & 1 & 4 & 9 \\
Number of sensor nodes & 50 & 50 & 50 \\
\hline
\end{tabular}

In the situation of terrain I, II and III, after the terrain correction, the coverage hole 
boundaries as shown in Figure 14, Figure 15 and Figure 16.

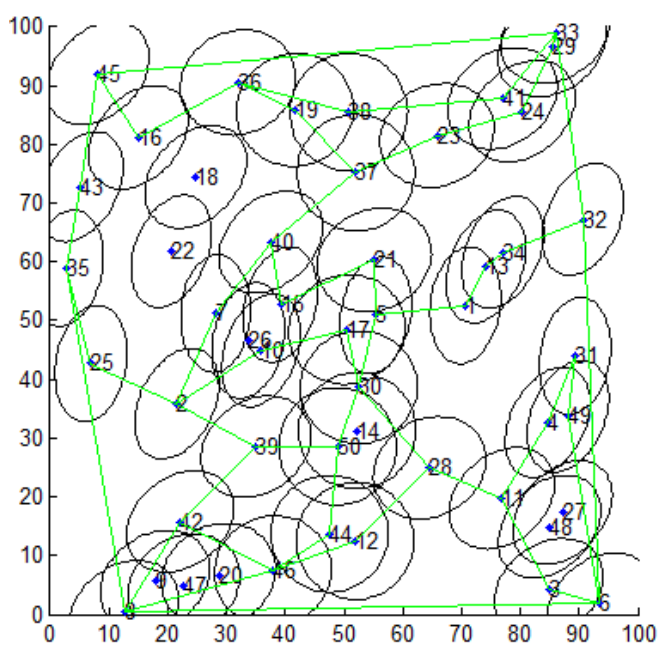

Figure 14. Terrain I Correction

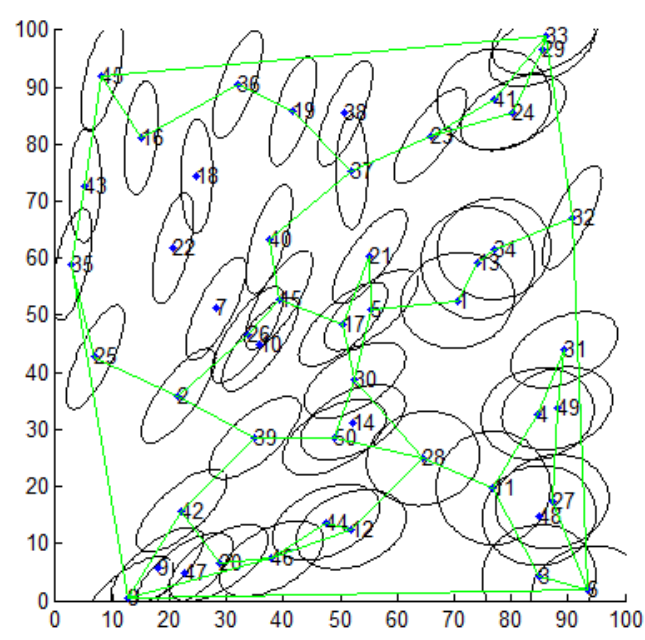

Figure 15. Terrain II Correction

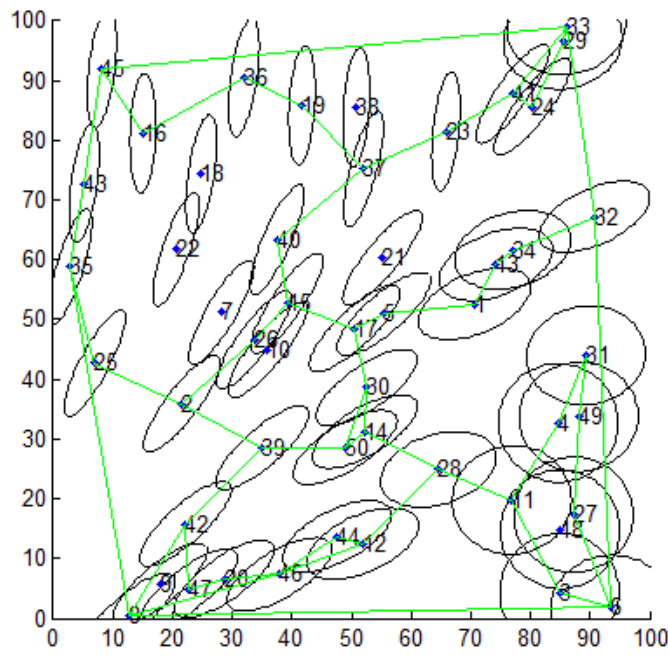

Figure 16. Terrain III Correction

In the same area range and the same number of nodes, with the increase of the number of peaks and valleys, the terrain undulation degree is increasing. Comparing with terrain I, II and III, as terrain undulating degree increases, the area of coverage holes is increasing. Then boundaries of coverage holes are increasing. Finally the coverage rate of WSN is decreasing. The simulation results show that it also applies to undulating obviously terrain.

In order to further evaluate the performance of three-dimensional terrain correction methods, under the same experimental conditions, compared with traditional correction method (Liu and $\mathrm{Fu}$ [8] proposed detection of trap coverage holes in WSNs based on topographic correction). The "flat" means the sensor node initial coverage rate, as shown in Figure 17. 


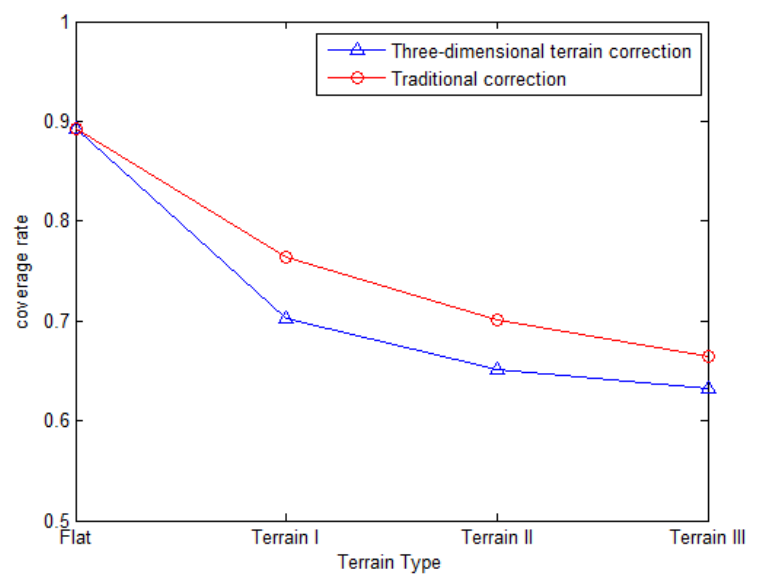

Figure 17. Compared with the Traditional Method

After terrain correction, the coverage rate of three-dimensional terrain correction and traditional correction are gradually decreased. Compared to traditional correction, the coverage rate of three-dimensional terrain correction is lower. It shows that three-dimensional terrain correction method can detect more coverage holes in the target area. Thus, three-dimensional terrain correction method is better for coverage hole detection than traditional correction. Three-dimensional terrain correction method is also more suitable for undulating obviously terrain.

\section{Conclusions}

For three-dimensional undulating terrain features, in this paper, we propose a detection method of coverage holes in wireless sensor networks based on three-dimensional terrain correction. Through the detection algorithm of coverage holes boundary, we obtain the coverage holes boundaries of the target area. Simulation results show that the boundaries of coverage holes become bigger. The results are also applicable to three-dimensional undulating obviously terrain. For the sensor nodes deployment of undulating terrain, it has reference value.

\section{Acknowledgements}

This work was supported by the National Natural Science Foundation of China (No. E050603), Guangxi Universities Scientific Research Projects (No. YB2014157), Guangxi Natural Science Foundation Program(No. 2015GXNSFBA139254).

\section{References}

[1] WANG Wei, LIN Feng, ZHOU Jiliu. Research Progress on Coverage Problem in Wireless Sensor Networks[J]. Application Research of Computers, vol. 27, no. 1, (2010), pp. 32-35.

[2] XU Chugui, DENG Xiaoheng, ZOU Haojie.Repair Policies of Coverage Holes in Wireless Sensor Networks[J]. Chinese Journal of Sensors and Actuators, vol. 23, no. 2, (2010), pp. 256-259.

[3] Hwa Chun M, Prasan Kumar S, Yen Wen C. Computational geometry based distributed coverage hole detection protocol for the wireless sensor networks[J]. Journal of Network and Computer Applications, no. 34, (2011), pp. 1743-1756.

[4] DAI Guoyong, CHEN Luyi, ZHOU Binbin, et al.. Coverage Hole Detection Algorithm Based on Voronoi Diagram in Wireless Sensor Network[J]. Journal of Computer Applications, vol. 35, no. 3, (2015), pp. 620-623.

[5] GAO Hao, WANG Qingsheng, FENG Xiufang, et al.. Coverage Blind Spots Discovery Algorithm in WSNs[J]. Transducer and Microsystem Technologies, vol. 31, no. 9, (2012), pp.109-115.

[6] XING Dongping, DUAN Fu, FAN Maosen. Coverage Blind Spot Discovery Algorithm for WSNs based on polar coordinate[J]. Transducer and Microsystem Technologies, vol. 33, no. 9, (2014), pp. 117-119. 
[7] LI Mingyi, CHEN Junjie. Coverage Holes Detection Algorithm in Wireless Sensor Networks. Computer Measurement \& Control, vol. 21, no. 9, (2013), pp.2501-2505.

[8] LIU Ye, FU Zhongqian. Detection of Trap Coverage Holes in WSNs Based on Topographic Correction[J]. Chinese Journal of Sensors and Actuators, vol. 27, no. 6, (2014), pp.785-790.

[9] Xiao Yang L, Kai Liang W, Yanmin Z, et al.. Mobility increases the surface coverage of distributed sensor networks. Computer Networks, no. 57, (2013), pp.2348-2361.

[10] LIU Zhikun, XIA Qingtao, LUO Hao, et al.. Research on Three-Dimensional Coverage Strategy for Wireless Sensor Networks[J]. Journal of Wuhan University of Technology(Transportation Science \& Engineering), vol. 37, no. 3, (2013), pp.581-584.

[11] LI Meng, DING Dairong, GUO Tingli. Random Deployment Strategy of Wireless Sensor Network Node[J]. Computer Engineering, vol. 38, no. 5, (2012), pp.99-101.

[12] XU Yixin, BAI Yan, ZHAO Tianyang, et al.. Multi-objective Coverage Control in Wireless Sensor Network Based on Poisson Distribution[J]. Journal of Computer Applications, vol. 33, no. 7, (2013), pp.1820-1824.

[13] GONG Jianya, LI Xiaolong, WU Huayi. Spatiotemporal Data Model for Real-time GIS[J]. Acta Geodaetica et Cartographica Sinica, vol. 43, no. 3, (2014), pp.226-232.

[14] HUANG Yue, XIANG Shu, XIAO Lei, et al.. Research Progress of The Node Deployment for Wireless Sensor Network [J]. Control Engineering of China, vol. 19, no. 4, (2012), pp.644-649.

[15] YU Jie, LV Pin, ZHENG Changwen. A Comparative Research on Methods of Delaunay Triangulation[J]. Journal of Image and Graphics, vol. 15, no. 8, (2010), pp.1158-1167.

[16] HAN Yuanli. A General-division Grid Pattern Delaunay-TIN Parallel Algorithm[J].Acta Geodaetica et Cartographica Sinica, vol. 44, no. 6, (2015), pp.702-708.

[17] Wei L, Wei Z. Coverage hole and boundary nodes detection in wireless sensor networks[J]. Journal of Network and Computer Applications, no. 48, (2015), pp.35-43.

[18] HU Zhongdong, XIE Jinwei. The 3D Localization Mechanism for Wireless Sensor Networks Based on Mountainous Terrain[J]. Chinese Journal of Sensors and Actuators, vol. 28, no. 3, (2015), pp.408-411.

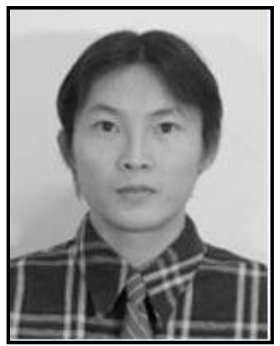

\section{Authors}

Shen xianhao received the Ph.D degree in Beijing Institute of technology. He is currently an associate professor in College of Information Science and Engineering in Guilin University of Technology. His research interests include wireless sensor networks and intelligent diagnosis.

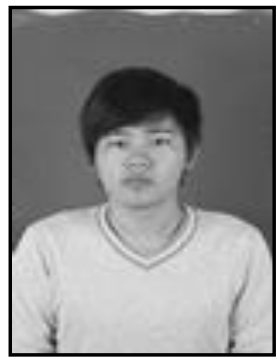

Li jun received his bachelor degree in Chaohu university. He is currently a graduate student in Guilin University of technology. His research interest is wireless sensor networks. 